\title{
Dose-dense AC Followed by Paclitaxel/Trastuzumab Regimen
}

National Cancer Institute

\section{Source}

National Cancer Institute. Dose-dense AC Followed by Paclitaxel/T rastuzumab Regimen. NCI Thesaurus. Code C138036.

A dose-dense chemotherapy regimen consisting of doxorubicin and cyclophosphamide followed by paclitaxel and trastuzumab, used as a neoadjuvant or adjuvant treatment for HER-2/neu-positive breast cancer. 